\title{
Controlling Next-Generation Software-Defined RANs
}

\author{
Arled Papa*, Raphael Durner*, Leonardo Goratti ${ }^{\dagger}$, Tinku Rasheed ${ }^{\dagger}$, Wolfgang Kellerer* \\ ${ }^{*}$ Chair of Communication Networks, Technical University of Munich \\ ${ }^{\dagger}$ Zodiac Inflight Innovations, Weßling Germany \\ *arled.papa@tum.de, *r.durner@tum.de, ${ }^{\dagger}$ leonardo.goratti@zii.aero, ${ }^{\dagger}$ tinku.rasheed@zii.aero, \\ *wolfgang.kellerer@tum.de
}

\begin{abstract}
Compared to previous mobile generations, the 5G system architecture offers more flexibility to fulfill a richer set of diverse requirements. Three system aspects play a major role here: support of network slicing, a split between the user plane and the control plane, and a service oriented control architecture. Emerging techniques of network softwarization such as SoftwareDefined Networking (SDN) are leveraged to provide this notion of flexibility manifested in a Software-Defined core network. However, in addition to the $5 \mathrm{G}$ Core (5GC), analogous concepts could be applied to the Next Generation Radio Access Network (NG-RAN) to sustain the required system performance. Driven by the increased attention that is currently paid to a SoftwareDefined Radio Access Network (SD-RAN), in this article, we propose the Radio Access Network Control Function (RANCF) as an additional 5G control function. We define the main functionalities of RANCF and propose interfaces and protocols for the interaction with 5GC functions. Exploiting network slicing as an example, we demonstrate how RANCF facilitates the slice selection and slice management procedure.
\end{abstract}

Index Terms-5G, Flexibility, Network Slicing, SD-RAN, RANCF.

\section{INTRODUCTION}

Software-Defined Networking (SDN) is paving the way towards programmable and flexible next generation networks. The novelty of 5G manifests in the flexibility to support heterogeneous applications based on new concepts such as network slicing, control and user plane decoupling and abstraction of the control plane functions in terms of a service based architecture [1].

Considering the heterogeneous requirements of emerging applications, network slicing is one of the key concepts of 5G. This implies the embedding of multiple network slices (i.e., shares of the network), which can be operated on top of the same physical infrastructure in an isolated fashion while guaranteeing Quality-of-Service (QoS) requirements [2]. Hence, network slicing leads to an increased overall network performance, considering a flexible support of heterogeneous applications by fulfilling different requirements in separate slices and multiplexing between them. Therefore, the concept of network slicing has been embraced by 3GPP in its proposed $5 \mathrm{G}$ architecture and new functions (i.e., network slice selection function) have been introduced to enable its support.

The decoupling of the control plane and the user plane provides a new level of interaction based on the concept of SDN. SDN suggests the centralization of the control functions in entities referred to as SDN controllers. So far this concept has mainly been addressed in the 5G Core (5GC) architecture of the current $5 \mathrm{G}$ standard.

In order to also bring the notion of SDN to NG-RAN, Software-Defined RAN (SD-RAN) controllers are envisioned to control eNBs/gNBs. More specifically, SDN in RAN has been shown promising in terms of improving resource management and efficiency in a domain where wireless resources are the bottleneck. On the one hand, the separation of control and user plane implied by SDN in RAN can provide a broader network view by centralizing the control functionalities in SD-RAN controllers. This can lead to enhanced coordinated scheduling and power management, which can further improve the network performance. On the other hand, such coordination induces higher complexity on the SD-RAN controllers and increases the control overhead, which can result in inability to support strict delay operations such as scheduling or random access procedure.

Several open source SD-RAN platforms have been proposed [3], [4], which address the concept of a softwarized control over the RAN. The main focus lies on radio resource management and provision of isolation for network slices. Recent works [5], [6] have proven that a coordination among the decisions of network slices' schedulers and an SD-RAN master controller can guarantee the required level of QoS, and at the same time improve the end-to-end network slicing efficiency. However, while the existing $5 \mathrm{G}$ architecture already provides an SDN-based control for 5GC, its RAN counterpart is missing. We think an analogous concept should be realized for the NG-RAN.

In this article we introduce the Radio Access Network Control Function (RANCF). RANCF corresponds to a realization of an SD-RAN controller in a 5G NG-RAN architecture. In principle, RANCF is envisioned for the control and coordination of multiple gNBs. In compliance with the 5G system architecture, RANCF is defined as a flexible and easy to integrate network function. From the network operators' and vertical stakeholders' perspective, RANCF alleviates the challenge of deploying and managing network slices by enabling a coordination mechanism between the NG-RAN and $5 \mathrm{GC}$, which is missing in the existing $5 \mathrm{G}$ architecture. Moreover, while controlling multiple gNBs, RANCF maintains a centralized network view that can increase radio resource efficiency with potential lower signaling overhead compared 


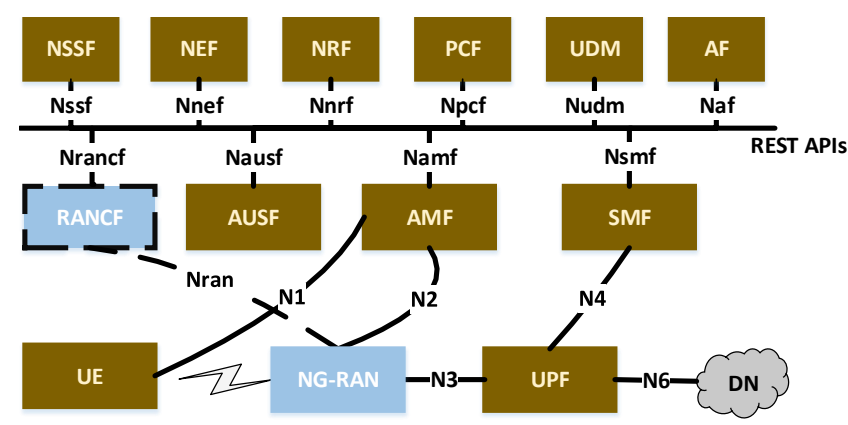

Figure 1: Envisioned 5G functional architecture based on the 3GPP Release 15 including the proposed RANCF function. NG-RAN functions are makred in blue and 5GC functions in brown. A breakdown of the NG-RAN functions is illustrated in Fig. 2

to distributed approaches. To enable this support, we define interfaces and protocols that can be used for the interaction of RANCF with existing 5G functions and shed light on its control functionalities. Finally, we leverage network slicing as a main 5G use case and show how RANCF acts for network slice selection, life cycle and management.

The rest of this article is organized as follows. Section II describes the overall 5G architecture and explains the main functionalities of the 5GC and NG-RAN, respectively. We introduce a programmable and softwarized 5G RAN architecture in Section III and propose RANCF. Section IV concerns network slicing and introduces the process of network slice selection and management in 5G. Finally Section V discusses the benefits and implications of RANCF, whereas Section VI concludes our work.

\section{5G ARCHITECTURE}

The overall 5G architecture of Release 15 [1] enhanced with our proposed RANCF is depicted in Fig. 1. It has two major parts namely 5G Core (5GC), which consists of the functions denoted by brown color in Fig. 1 and Next-Generation Radio Access Network (NG-RAN) whose functions are denoted by light blue. In this section, we describe in more detail the main $5 \mathrm{G}$ functions of both $5 \mathrm{GC}$ and NG-RAN. In particular, we elaborate the process of QoS handling in 5G and highlight potential improvements of the proposed RANCF.

\section{A. 5GC Network Functions}

Taking as start the existing 4G Core architecture, the main functionalities of the Mobility Management Entity (MME), Service/Packet Gateways (S/P GW), Home Subscriber Server (HSS) and Policy and Charging Rules Function (PCRF) are kept in the 5GC and distributed over multiple functions decoupling user and data planes. Additionally, functions with respect to network slicing and applications support are introduced. The overall 5GC functions are as follows:

1. Authentication and Mobility Function (AMF): responsible for Non-Access Stratum (NAS) (i.e., manages the establishment and maintenance of communication with the user equipment), as well as security and mobility handling.

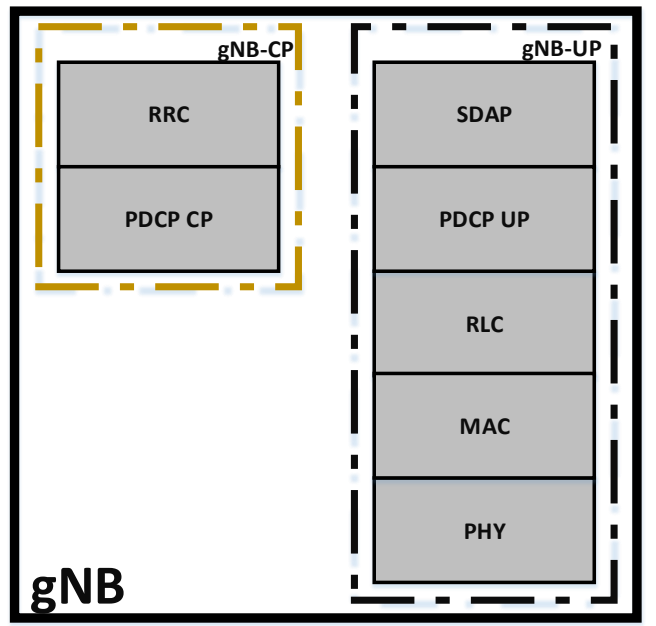

Figure 2: Overview of NG-RAN functions mainly realized in the $5 \mathrm{G}$ gNB (base station). We distinguish between control plane (gNB-CP) and user plane (gNB-UP) functions. Interfaces to the 5GC functions are shown in Fig. 1

2. User Plane Function (UPF): responsible for mobility anchoring and packet handling. It can further facilitate the packet forwarding, routing and inspection.

3. Session Management Function (SMF): responsible for user equipment IP address allocation and selection of the appropriate UPF for each network slice.

4. Unified Data Management (UDM): database that stores all the information concerning the subscribers of the network.

5. Authentication Server Function (AUSF)- carries user authentication functionalities.

6. Policy Control Function (PCF): responsible for applying network policies.

7. Network Exposure Function (NEF): responsible for exposing the capabilities of the network functions to candidate applications or third parties. The range of exposure can consist of monitoring, provisioning and traffic routing.

8. Network Slicing Selection Function (NSSF): facilitates the selection of the network slices.

9. Network Repository Function (NRF): responsible for advertising Network Functions (NFs) with management tasks such as registration, de-registration, authentication, discovery.

10. Application Function (AF): responsible for handling various applications that can be offered to the network.

\section{B. NG-RAN functions}

The overview of the NG-RAN is depicted in Fig. 2. The main entity of the NG-RAN is the gNB. Within the gNB, there exist several functionalities which we detail as follows:

1. Radio Resource Control (RRC): responsible for handling RAN control plane functionalities such as connection setup/release, control steering to 5GC (i.e., AMF), AMF selection upon UE arrival, mobility control, broadcasting of system information to UE (i.e., Signal Radio Bearers (SRB)), radio admission control. 
2. Service Data Application Protocol (SDAP): mainly responsible for the conversion of QoS flows to Data Radio Bearers (DRB).

3. Packet Data Convergence Protocol (PDCP): responsible for tasks including IP header compression, retransmissions and handling packet duplications during handovers.

4. Radio Link Control (RLC): in charge of packet segmentation tasks.

5. Medium Access Control (MAC): its responsibilities lie in the tasks of scheduling and logical channel multiplexing.

6. Physical Layer (PHY): it handles coding/decoding as well as modulation/demodulation tasks.

The 3GPP NG-RAN architecture [7] does not only distinguish between user plane and control plane functions, but also proposes a functional split for a physical separation of gNB functions between those residing in distributed units and functions in a centralized unit of NG-RAN. This functional split is envisioned to provide flexibility and enable radio resource coordination.

\section{Quality-of-service handling}

The advanced requirements for $5 \mathrm{G}$ in terms of its application heterogeneity demands for enhanced QoS mechanisms compared to $4 \mathrm{G}$. In $5 \mathrm{G}$ only the $5 \mathrm{GC}$ is aware of the QoS requirements, whereas the RAN is unaware of the service [8]. Each new UE connected to the network enables the initiation of one or more Packet Data Unit (PDU) sessions. In turn, a PDU session is assigned one or more QoS flows and Data Radio Bearers (DRBs). IP packets are converted into QoS flows in the UPF of the 5GC depending on data rate or delay requirements and then transported to the RAN. In the RAN the SDAP layer is responsible to enforce the QoS flow to DRB mapping. SDAP is a new introduced functionality of NG-RAN in order to sustain the essential requirement of QoS provisioning of $5 \mathrm{G}$ towards the entire network.

Although SDAP is the envisioned $5 \mathrm{G}$ function to handle the QoS flow to DRB mapping within one gNB, a concrete realization of this procedure remains open to implementation. Considering the aforementioned characteristics of the 5G network a QoS coordination mechanism among gNBs is necessary to provide an overall improved performance. We argue that the RANCF proposed in this article can be envisioned as the connection point between NG-RAN and 5GC and moreover can facilitate the radio resource coordination and network slicing among multiple gNBs.

\section{Control of Software-Defined RANs}

The application of the SDN concept to the $5 \mathrm{G}$ architecture manifests in the decoupling of control plane and user plane functions. While this principle is well established in the 5GC, in NG-RAN control and user plane functions are mainly residing in the gNB as depicted in Fig. 2.

With network slicing arising as a main feature of $5 \mathrm{G}$, there has been a vast amount of proposals to enable the control of network slices in the RAN via SD-RAN controllers. The main approach suggests a hierarchical scheduling decision in

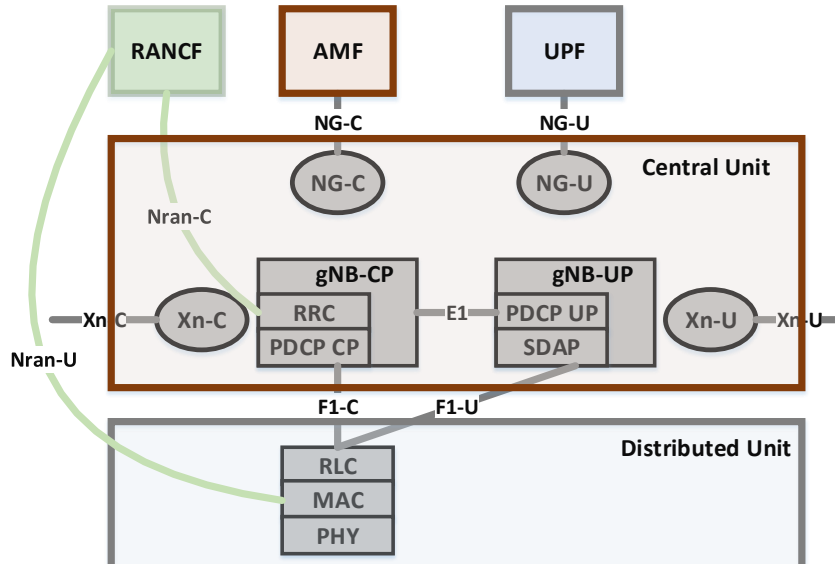

Figure 3: Envisioned NG-RAN functional architecture based on a separation of functions between distributed units, centralized units and an SD-RAN controller (RANCF). NG-C and NG-U are interfaces to the 5GC. Proposed interfaces to the proposed RANCF are Nran-C and Nran-U.

the RAN, where a master scheduler (i.e., SD-RAN controller) steers the resource allocation over multiple slice schedulers, which are in charge of scheduling decisions within a network slice [5], [6].

In the context of providing an SD-RAN controller within the 5G architecture, we propose the Radio Access Network Control Function (RANCF). RANCF will be mainly responsible for the control of the NG-RAN handling decisions such as scheduling, handover, power management. Therefore, in our envisioned 5G architecture shown in Fig. 1, RANCF is highlighted as a new $5 \mathrm{G}$ control function. Moreover, we define the Nran interface needed for the interaction of RANCF with the underlying RAN infrastructure as well as Nrancf, which is needed for RANCF's interaction with other existing 5GC functions (i.e., AMF, SMF, AF). A detailed description of the aforementioned functionalities, protocols and interfaces is provided in the remainder of this section.

\section{A. RANCF interaction with NG-RAN functions}

As aforementioned, 3GPP proposes a functional split within a gNB. Whereas several different RAN function splits are possible, we focus on the RLC-PDCP function split proposed in [7] as a typical example. However, any function split can be supported by RANCF. For the RLC-PDCP function split, in the distributed unit (i.e., gNB-DU), functions such as RLC, MAC and PHY are located, whereas PDCP and RRC functions are located in the so called central units (i.e., gNB-CU). Within the central unit, there is a logical separation between the control and the user plane of the gNB referred to as gNB-CU-CP for the control plane and gNB-CU-UP for the user plane, respectively. The connection between the gNB-CU and gNB$\mathrm{DU}$ is realized by the F1 interface, which in turn is separated in F1-C for control plane and F1-U for user plane. Likewise the connection between the gNB-CP and UP is realized by the E1 interface. In a similar fashion, the RAN control and user plane are connected to the $5 \mathrm{GC}$ control and user plane by utilizing NG-C and NG-U, accordingly. Finally, the RAN domain can 
contain multiple gNBs interconnected via the $\mathrm{Xn}-\mathrm{C}$ and $\mathrm{Xn}-\mathrm{U}$ interfaces.

In the above described $5 \mathrm{G}$ architecture, each $\mathrm{gNB}$ is responsible for the control of its user plane. There exists a communication among gNBs, however the main purpose is to facilitate the handover procedure. Such a distributed approach might lead to a suboptimal solution and furthermore it may increase the signaling overhead if additional tasks such as network slice scheduling and power management are added. In contrast, in our proposed architecture, the control over multiple gNBs is centralized in RANCF. In that regard, due to a broader view of the network RANCF can take smarter decisions and therefore boost the network performance, but also potentially reduce signaling overhead since only the affected gNBs will be involved by RANCF.

Following the logic of this architecture, we define the interfaces of RANCF with the appropriate functions in the gNB and illustrate our envisioned concept in Fig. 3. As described, the functionality of the RANCF is mainly to provide a centralized control of the RAN domain realizing an SD-RAN controller. This control for example may influence scheduling decisions, power allocation, modulation and coding schemes of specific gNBs. The gNB function in charge of the aforementioned tasks is the MAC. Hence we believe that a control connection between the RANCF and the MAC layer of the distributed unit of the gNB should be established. This connection is depicted in Fig. 3 as Nran-U. Similarly RANCF, can be utilized to coordinate decisions of the control plane of the gNB, which is located in the central unit. These control decisions may involve but are not limited to altering RRC decisions on the QoS management, handover decisions, establishment and modification of Signaling Radio Bearers (SRBs) and Data Radio Bearers (DRBs). Hence, in our proposed concept we envision a control communication between RANCF and RRC namely Nran-C. Both Nran-U and Nran-C denoted by Nran in Fig. 1, can be for instance protocols utilized by SD-RAN controllers such as the FlexRAN protocol [3].

\section{B. RANCF interaction with 5GC network functions}

While in the core part of the $5 \mathrm{G}$ architecture, SMF is responsible for the control decisions, for instance selecting the appropriate UPF for network slices, in the RAN part of the 5G architecture, the RANCF will be responsible for such tasks. It is therefore crucial that SMF and RANCF share a view of the network with each other in order to provide end-to-end guarantees. As illustrated in Fig. 1, control information between SMF and RANCF can be exchanged by using REST-like protocols. Moreover, RANCF should maintain communication with other control functionalities of 5GC, e.g., with AMF to gather information about UE mobility. Also here, REST APIs can be used. RANCF can also support diverse application requirements in the RAN. Specific requirements may even imply changes of the scheduling decisions or handovers. In such cases, the AF can utilize the Nran interface and apply application specific policies directly to RANCF using REST API and in turn RANCF should translate these policies to a format that is understood within the RAN.

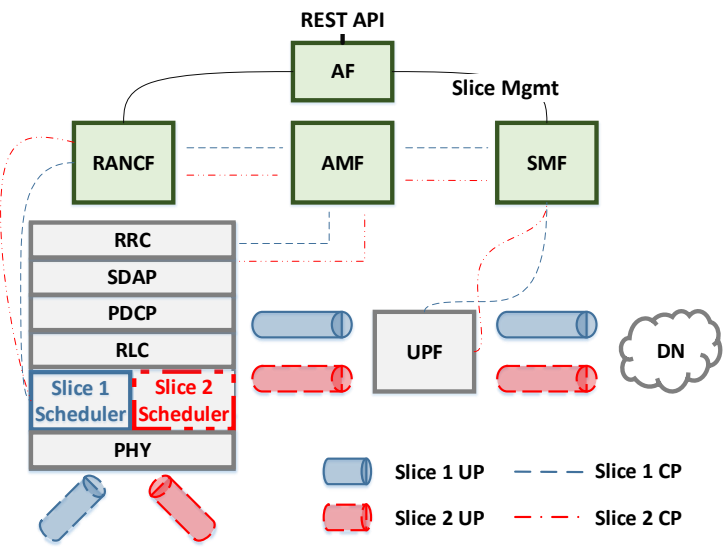

Figure 4: Illustration of the interaction of $5 \mathrm{G}$ functions and RANCF to support end-to-end network slicing. Dashed lines show control information flows. Tube shapes represent isolated user plane slices from the UE through the 5G RAN and core to the Data Network (DN).

\section{Network SLiCing IN 5G SUPported By RANCF}

Network slicing is considered a main feature of 5G. According to $5 \mathrm{G}$ information models [9], the implementation of a network slice is referred to as Network Slice Instance (NSI). An NSI can consist of multiple Network Slice Subnet Instances (NSSIs). Namely, an NG-RAN NSSI and a 5GC NSSI, each of them having Network Functions (NFs) to meet the QoS requirements of the NSI. In this section we elaborate more on the concept of network slicing with respect to the state of the art and in 3GPP standardization. In particular, we use the example of network slicing to illustrate the role of RANCF in the 5G architecture.

\section{A. Network slicing in Core Network}

Network slicing in the core network side has been a hot topic for many years. The main concept manifests in enabling the co-existence of multiple network slices on the same physical infrastructure by guaranteeing the required level of QoS. In principle this problem is tackled by differentiating the IP packets of specific services and mapping them to appropriate QoS flows. Core network switches take care of handling the QoS flow forwarding to achieve the related throughput or delay guarantees. 3GPP in its Release 14 has already standardized this approach under the name evolved DECOR (eDECOR) [10].

\section{B. Network Slicing in RAN}

While network slicing has been mainly applied in the core network side, RAN slicing has emerged as a complementary approach to boost network efficiency. As a result, research towards identifying efficient RAN slicing solutions has been initiated.

In [11] four possible RAN slicing multiplexing options are presented. The difference among the options lies in the tradeoff between isolation and multiplexing gain. The proposed solution suggests the share of the spectrum among the slices, 
which results in a more dynamic and therefore efficient implementation. Nonetheless this option renders isolation preservation more challenging.

\section{End-to-end Network Slicing}

As earlier mentioned in this paper, $5 \mathrm{G}$ applications require for stricter isolation guarantees due to stringent delay requirements. The question then arises how such guarantees can be assured.

State of the art proposals answer this question by introducing possible solutions for end-to-end network slicing. [12] proposes a flow-based network slicing approach. Both RAN and core are controlled by an SDN-like controller. Whereas, the communication between the RAN and the core has to surpass an Open vSwitch. Slices are created by the SD-RAN controller. Further the slice information is sent to the core controller which in turn deploys the appropriate flow rules on the Open vSwitch. On the other hand [13] introduces an endto-end slicing approach to enable low latency edge applications in the network. It also utilizes Open vSwitch to steer the information towards an edge computation platform to cater for delay reductions for specific sensitive applications. Both proposals provide viable solutions for coordinating network slices in the core as well as in the RAN part. Yet a concrete mapping and embedding of such solutions to the $5 \mathrm{G}$ architecture standard is missing.

Driven by existing solutions in the literature, we depict our view of the end-to-end network slicing concept in Fig. 4. We argue that a coordination among the NG-RAN NSSI and 5GC NSSI is necessary to enhance network performance, since a lack of coordination cannot achieve the required level of isolation of the NSI. For instance, if the operator supports network slicing only in the RAN, there is no guarantee that the QoS can be preserved if not appropriate UPFs are reserved in the core. Likewise, if only the core is slice aware, there is no guarantee that the required amount of radio resources associated with each network slice can be granted. For each deployed network slice apart from time and frequency resources in the RAN, appropriate backhaul bandwidth should be reserved in the tunnel between the RAN and UPF. Hence, the control coordination among RAN and core becomes mandatory. In the following, we map the SDRAN concept manifested in the proposed RANCF in analogy with the already developed open source platforms [12], [13] for an end-to-end slicing solution to the current $5 \mathrm{G}$ functional architecture. SMF can be understood as an SDN controller of the core network, whereas RANCF resembles the SDRAN controller. An east/west communication API is therefore needed for control and coordination. The REST API envisioned for the communication amongst the functions in the 5G core presented in Fig. 1 can be used for that purpose. In that regard, a protocol is needed for control information exchange. The protocol shall support the 5G information model known as Network Resource Model (NRM) based on XML or JSON formats. Using such data models, information between the NG-RAN NSSI and 5GC NSSI can be exchanged with respect to slice resource utilization. In this way an end-to-end flow control of the NSI can be maintained.

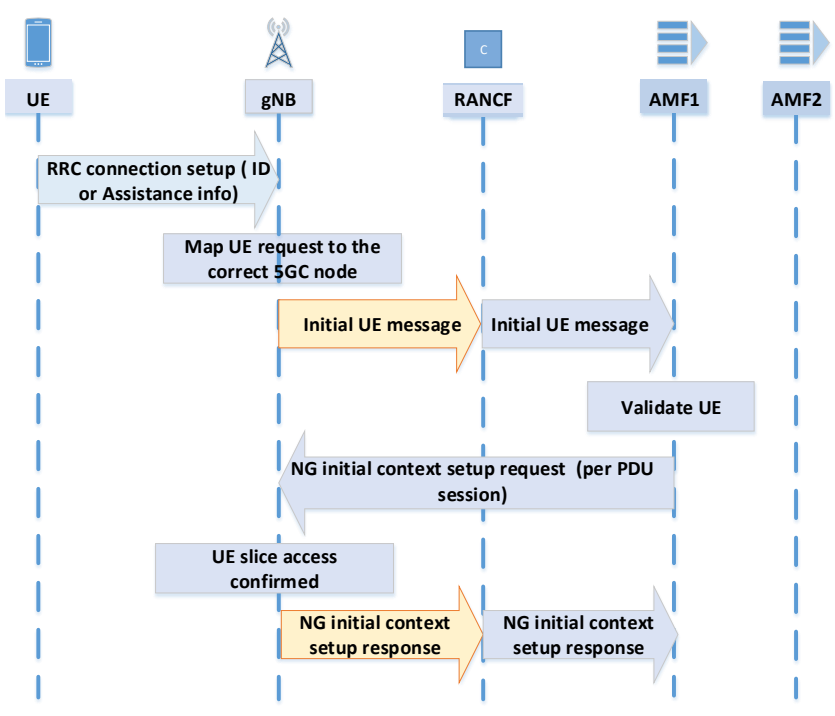

Figure 5: Network slice selection signaling in 5G.

\section{Network Slice Creation \& Management}

The creation and management of network slices raises many questions and remains an important topic for research. In the following we propose an answer to the open questions regarding network slice creation/management using our proposed RANCF and based on recent 3GPP studies [9].

From the logical point of view, a network slice is requested by a service or a network operator. Therefore, the slice creation is offloaded to the application function (AF) in 5G. We believe that the $\mathrm{AF}$ will be in charge of the network slice management coordination process and slice acceptance admission. It should be able to gather information from both core and RAN part of the $5 \mathrm{G}$ network to sustain a global view and act depending on the availability of both SMF and RANCF. Whereas, decisions on the amount of UEs in a slice are then part of the admission control of individual network slices. In our proposed 5G architecture, the deployment part of the NSI is thus a task of the application functions. As it is illustrated in Fig. 4 the service can be part of the same operator or requested by another operator or service provider out of the operator's domain by utilizing a REST API.

\section{E. Network slice selection}

While network slice creation and management is still unclear, 3GPP in Release 15 [1] has already standardized the slice selection procedure. Initially each AMF connected to the RAN is requested to identify the list of slices that they can support. This request is referred to as Next-Generation (NG) setup request. Each UE can select the preferred network slice to attach to by including an identification (ID) or an assistance information. This information is then utilized by the RAN in order to steer the UE request to the appropriate AMF. The AMF then validates the UE by requesting information from the Network Slice Selection Functions (NSSF), whether the UE is allowed to attach to the requested slice. If the UE is successfully attached, SMF selects the appropriate UPF for 
the UE and initiates a Packet Data Unit (PDU) session. The RAN is then notified by receiving a NG context setup request. It is then the RAN's duty to perform resource allocation for a successful transmission to be initialized. Since the RANCF is responsible for scheduling decisions for network slices, it should be notified for all the slices that are created, as well as the users of each network slice. This means that all the slice initiation/termination information of users should be noted by the RANCF in order to have a clear view of the network. Thus, in our view the 3GPP slice selection procedure should involve RANCF as depicted in Fig. 5.

\section{DISCUSSION}

We have emphasized the important role of RANCF in a 5G architecture and highlighted its interaction with existing 5G core and RAN functions to fulfill the network slicing concept, in particular, proposing concrete protocols and interfaces. Moreover, we showed the importance of RANCF from the network operators' and verticals' perspective that lies in relieving the management and orchestration challenge of a network slice. In light of the benefits that RANCF brings to $5 \mathrm{G}$, also the potential overhead has to be taken into account, mainly from additional signaling that is introduced.

Intuitively, the signaling overhead is affected by the number of slices, users of a slice and gNBs that the RANCF is controlling as well as the size and frequency of control message updates. Initial benchmark of FlexRAN SD-RAN controller is provided in [14], where the impact of controlling multiple gNBs is presented with respect to memory, CPU consumption and slice initiation time. Even though users are not present in the current version of the benchmarking tool, an insight of signaling overhead is provided for the impact of the underlying network on SD-RAN controllers. This benchmarking tool can be considered as an initial implementation of RANCF and can be further extended to enable the communication with $5 \mathrm{GC}$ functions and establish the management of the end-toend network slicing.

The key performance indicators (KPIs) of RANCF to compare it with other proposed solutions or to evaluate its efficiency revolve around slice life-cycle KPIs such as slice deployment time, slice scalability and slice reconfiguration time as elaborated in [15]. Furthermore, slice efficiency KPIs extend also to radio (e.g., bandwidth) and mobile core (e.g., backhaul bandwidth, CPU cores, memory) resource utilization.

From a standard's perspective, RANCF could be easily integrated into the $5 \mathrm{G}$ architecture. That means there are no particular changes to the $5 \mathrm{G}$ architecture required. Since with RANCF we follow the logic of a service oriented architecture as proposed for $5 \mathrm{G}$, we introduce our proposed RANCF as a new function. We utilize the existing REST protocol proposal for the envisioned required communication. Hence, we infer that our function can be easily adopted by the $5 \mathrm{G}$ architecture with minimum extra effort.

\section{CONCLUSION}

5G networks have been envisioned to accommodate the enormous growth in traffic requirements and the heterogeneity of user applications. In that regard several new concepts have been introduced in $5 \mathrm{G}$ to allow for this level of flexibility. The $5 \mathrm{G}$ architecture is driven by the concept of virtualization of network functions, which brings the required level of flexibility and programmability to the network. In particular for the concept of network slicing, new network functions have been introduced to support the deployment of slices in the network. In order to further enhance the programmability and flexibility in the RAN part of the $5 \mathrm{G}$ architecture, in this paper we propose a new control function namely Radio Access Network Control Function (RANCF). RANCF leverages the recently introduced concept of SD-RAN and allows for several functionalities in the network such as scheduling decision, power management and QoS handling support. Moreover, we elaborate on the interaction of RANCF with other existing $5 \mathrm{G}$ network functions and illustrate its importance and show how RANCF can coexist in the 5G architecture and facilitate important tasks related to network slicing.

\section{REFERENCES}

[1] 3GPP, "TR 21.915 V1.1.0 (2019-03); Technical Report; Summary of Rel-15 Work Items (Release 15)."

[2] N. Alliance, "Description of network slicing concept," NGMN $5 G P$, vol. 1, 2016.

[3] X. Foukas, N. Nikaein, M. M. Kassem, M. K. Marina, and K. Kontovasilis, "FlexRAN: A flexible and programmable platform for softwaredefined radio access networks," in Proceedings of the 12th International on Conference on emerging Networking EXperiments and Technologies, 2016.

[4] E. Coronado, S. N. Khan, and R. Riggio, "5G-EmPOWER: A SoftwareDefined Networking Platform for 5G Radio Access Networks," IEEE Transactions on Network and Service Management, 2019.

[5] A. Papa, M. Klugel, L. Goratti, T. Rasheed, and W. Kellerer, "Optimizing dynamic RAN slicing in programmable 5G networks," in IEEE International Conference on Communications (ICC), 2019, pp. 1-7.

[6] C.-Y. Chang, N. Nikaein, and T. Spyropoulos, "Radio access network resource slicing for flexible service execution," in IEEE Conference on Computer Communications Workshops (INFOCOM), 2018.

[7] 3GPP, "TS 38.401 V15.6.0 (2019-07); Technical Specification Group Radio Access Network; Architecture description (Release 15)."

[8] E. Dahlman, S. Parkvall, and J. Skold, 5G NR: The next generation wireless access technology. Academic Press, 2018.

[9] 3GPP, "TR 28.801 V15.1.0 (2018-01); Technical Report; Study on management and orchestration of network slicing for next generation network (Release 15)."

[10] 3GPP, "TR 23.711 V14.0.0 (2016-09); Enhancements of Dedicated Core Networks selection mechanism (Release 14)."

[11] S. E. Elayoubi, S. B. Jemaa, Z. Altman, and A. Galindo-Serrano, "5G RAN slicing for verticals: Enablers and challenges," IEEE Communications Magazine, vol. 57, no. 1, pp. 28-34, 2019.

[12] E. Coronado and R. Riggio, "Flow-Based Network Slicing: Mapping the Future Mobile Radio Access Networks," in 2019 28th International Conference on Computer Communication and Networks (ICCCN). IEEE, 2019, pp. 1-9.

[13] N. Nikaein, X. Vasilakos, and A. Huang, "LL-MEC: Enabling low latency edge applications," in 7th International Conference on Cloud Networking (CloudNet). IEEE, 2018.

[14] A. Papa, R. Durner, F. Edinger, and W. Kellerer, "SDRBench: A Software-Defined Radio Access Network Controller Benchmark," in IEEE NetSoft-3rd Workshop on Performance Issues in Virtualized Environments and Software Defined Networking (PVE-SDN), 2019, pp. 3641.

[15] S. Kukliński and L. Tomaszewski, "Key Performance Indicators for 5G network slicing," in IEEE NetSoft-2nd International Workshop on Advances in Slicing for Softwarized Infrastructures (S4SI), 2019, pp. 464-471. 
Arled Papa is a Ph.D. student since 2018 at the Chair of Communication Networks, Technical University of Munich, Germany, where he also received his Master of Science in Communications Engineering with high distinction in 2017. His research focuses on the design and analysis of QoS, network slicing and network virtualization concepts in aeronautical applications, inflight entertainment and connectivity services.

Raphael Durner is a Ph.D. student at Technical University of Munich, Germany, where he also received his Masters degree in 2014. His research focuses on network softwarization approaches and their impacts on network performance.

Leonardo Goratti received his Ph.D. degree in wireless communications in 2011 from the University of Oulu, Finland, and his M.Sc. in telecommunications engineering in 2002 from theUniversity of Firenze, Italy. He is currently part of research staff at Safran S. A.
Tinku Rasheed is a senior research staff member at Safran S. A. He has extensive industrial and academic research experience in the areas of mobile wireless communication and data technologies, end-to-end network architectures and services. He has several granted patents and has published his research in major journals and conferences.

Wolfgang Kellerer (M96SM11) is a Full Professor with the Technical University of Munich (TUM), heading the Chair of Communication Networks at the Department of Electrical and Computer Engineering. Before, he was for over ten years with NTT DOCOMO's European Research Laboratories. He received his Dr.-Ing. degree (Ph.D.) and his Dipl.-Ing. degree (Master) from TUM, in 1995 and 2002, respectively. His research resulted in over 200 publications and 35 granted patents. He currently serves as an associate editor for IEEE Transactions on Network and Service Management, IEEE Networking Letters and on the Editorial Board of the IEEE Communications Surveys and Tutorials. He is a member of ACM and the VDE ITG. 